ecological benefits, as Gordon et al. suggest, could usher in better public acceptance of bioengineering approaches to species conservation: with the International Union for the Conservation of Nature forecasting ${ }^{6}$ that up to $50 \%$ of all tree species in Europe alone will be extinct by 2050 , the potential for species rescue, such as in the case of the American chestnut ${ }^{7}$, deserves considerable attention.

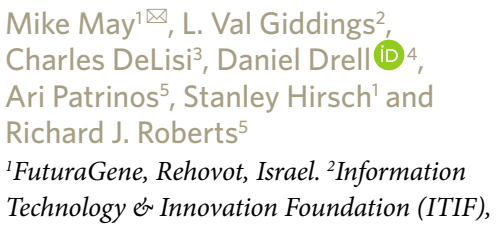

Washington, DC, USA. ${ }^{3}$ College of Engineering, Boston University, Boston, MA, USA. ${ }^{4}$ NOVIM, Santa Barbara, CA, USA. ${ }^{5}$ New England Biolabs, Ipswich, MA, USA.

$凶_{e-m a i l: m i k e @ f u t u r a g e n e . c o m}$

Published online: 6 January 2022 https://doi.org/10.1038/s41587-021-01189-8

\section{$\square$}

4. Danish Council on Ethics. Statement on GMO and ethics in a new era. https://www.etiskraad.dk/ /media/Etisk-Raad/en/ Publications/DCE_Statement_on_GMO_and_ethics_in_a_ new era 2019.pdf? la $=$ da (2019).

5. Foote, N. German Green faction pushes for gene editing, overhaul of regulation. Euractiv https://www.euractiv.com/section/ agriculture-food/news/german-green-faction-pushes-forene-editing-overhaul-of-regulation/ (June 2020).

6. International Union for the Conservation of Nature. Over half of Europe's endemic trees face extinction. https://www.iucn.org/ news/species/201909/over-half-europes-endemic-treesface-extinction (September 2019)

7. Melton, L. Nat. Biotechnol. 39, 400 (2021)

R.J.R works for New England Biolabs, a company that sells research reagents, including restriction enzymes and DNA MTases, to the scientific community. M.M and S.H work for FuturaGene Ltd., a company that employs a number of approaches towards tree improvement, including genetic modification.

\title{
The DxConnect Virtual Biobank connects diagnostic researchers to clinical samples
}

To the Editor - The first molecular tests for COVID-19 were ready for action in Europe a little over a month from the declaration of the pandemic. Similarly, the timeline for the more complex task of developing high-quality rapid antigen tests was compressed into just 8 month $^{1}$ - in comparison, development of the first rapid test for HIV took 5 years. Even so, from early on in the pandemic, a critical bottleneck in the development of reliable diagnostic tests was the scarcity of clinical samples for diagnostic research, development and validation. As pointed out in your June 2020 editorial, not only was there a shortage of specimens available for research, but scientific progress was hampered by the complexity of both negotiating legal agreements for sample transfer and jurisdictional regimes regulating sample transport across borders. Here, we describe the DxConnect Virtual Biobank (https:// www.finddx.org/biobank-services/vbd/), which acts as a clearinghouse for specimens, connecting researchers with institutions around the world that have clinical samples available for the development and validation of new diagnostic tests (Fig. 1).

Biological samples are critical for the development and validation of all new diagnostic tests. Yet we saw that even small quantities of SARS-CoV-2 samples only started to become available from commercial biobanks in around June 2020, and these were often limited not only in terms of clinical attributes (mainly from patients with ongoing, symptomatic infections), but also in geographic representation (mainly from Europe and the United States). Diagnostic companies were obliged to set up their own sample collections through ad hoc clinical trials. The result was little information on the performance of new tests outside of specific contexts of use, viral strains or populations in which they could work best.

COVID-19 is an example in which the eyes of the entire world were focused on one single disease, and still sharing proved difficult. No wonder then that accessing high-quality, well-characterized samples for the swath of existing, yet under-resourced, deadly diseases (for example, malaria, tuberculosis, hepatitis $\mathrm{C}$ and neglected tropical diseases (NTDs)) in low- and middle-income countries (LMICs) remains a complex problem.

Those working in diagnostic R\&D cannot develop new tests without efficient access to fit-for-purpose samples. To develop a new diagnostic test and validate its performance in intended clinical and epidemiological settings, biological samples should come from the concerned populations and geographies. Although the infectious diseases most in need of new diagnostic tests tend to occur in LMICs, most biobanks are located and operate in Europe and North America $^{2}$ and contain relatively few sample collections from LMIC settings.

Samples are often (and in LMICs, usually) collected by multiple local institutions, such as research institutes, hospitals and universities. Since these entities commonly work in isolation with limited networking and coordination tools, samples that could be greatly beneficial to R\&D may be invisible to those who need them, even if they are situated in the same country.

When these locally collected samples are discoverable by international $\mathrm{R} \& \mathrm{D}$ teams, they may be shipped, stored and used elsewhere. In some cases this can deprive local research opportunities of these samples. Ethical questions are also raised when the results of research do not benefit the populations whose samples were integral to that research. Transparency and equity in the collection, distribution and use of human specimens are not new themes; they have regularly resurfaced during previous health crises, including in Indonesia during the avian flu outbreak ${ }^{3}$ and during the Ebola epidemic that swept across Africa in 2014 (ref. ${ }^{4}$ ). Ensuring that LMICs can benefit from biomedical research, especially when they have contributed to its advancement, is paramount - this is the basis for the United Nations' Nagoya Protocol Access and Benefit Sharing (ABS) regime, which asserts the sovereignty of nations over biospecimens.

Lack of global standardization on the data used to characterize disease specimens is a further challenge to developers seeking samples - the content and provenance of the data provided can vary greatly from institution to institution, making it hard for developers to assess the data accuracy and to use samples from multiple sources.

These combined factors make it difficult for many people working in $R \& D$ to locate and access the samples they 


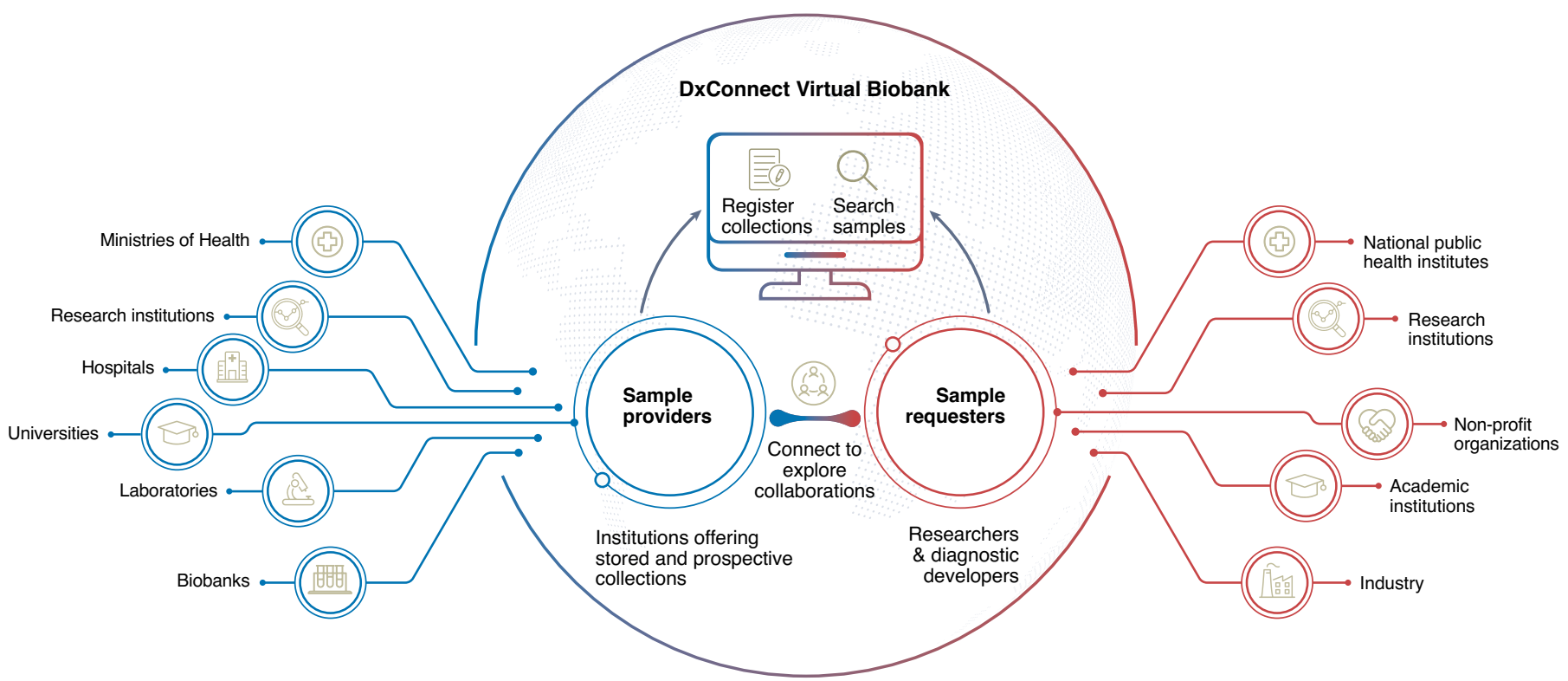

Fig. 1 An open access resource connecting diagnostic developers to clinical samples. The platform provides a global view of infectious disease sample collections available from worldwide institutions.

need. Virtual biobanks have emerged as a viable option to boost visibility of and access to samples ${ }^{2}$. As electronic databases, they provide a global view of aggregate or individual standardized data on sample collections from dispersed institutions, irrespective of where the samples are physically stored.

The DxConnect Virtual Biobank is one such collaborative resource. Hosted by FIND, the global alliance for diagnostics, this open-access platform enables researchers across academic, non-profit and industry sectors to view collections registered by any institution worldwide, search by disease and other characteristics, and connect with those holding samples of interest. This includes institutions that do not formally operate as 'biobanks', which may not otherwise make their sample collections visible and/ or available. Throughout the process, institutions maintain full control over their samples and the terms of access.

The COVID-19 pandemic has been a catalyst for operationalizing the DxConnect Virtual Biobank, which already includes access to 12 COVID-19 collections from registered institutions in eight different countries. Combined, these collections feature over ten sample types, including swabs, saliva, serum and plasma, collected from $>8,000$ participants. In September 2021, the first NTD collections were added - an area that has been prioritized in recognition of the fact that, since the start of the COVID-19 pandemic, more diagnostic tests have been developed for COVID-19 than for all 20 NTDs in the past 100 years ${ }^{5}$.
However, as a disease-agnostic platform, the DxConnect Virtual Biobank is not limited in scope to samples from these diseases. Future efforts will focus on adding collections for other priority diseases for global health, such as tuberculosis and malaria.

Diagnostic development needs to constantly evolve in line with the exploration of new scientific avenues and technologies, and the detection of new pathogens. As the COVID-19 pandemic and other recent epidemics have shown, the effectiveness of any response is dependent on solid research collaborations and the relationships between biobanks, researchers, hospitals and the private and public sectors that enable such collaborative work ${ }^{6,7}$.

Development and validation of better tests depends on accessible, high-quality, well-characterized disease samples. The challenges to accessing the samples needed to develop and validate the performance of diagnostic tests are compounded by additional factors including the 'my sample' syndrome, limited funding, high levels of bureaucracy, technical hurdles, discrepancies in legislation and consent requirements between countries, and import and export restrictions ${ }^{8,9}$. Although the procurement of biological samples is thus not the only challenge facing the development of new diagnostic tests, virtual biobanks are emerging as a valuable means to ease this critical part of the process.

The DxConnect Virtual Biobank offers the global-health community a collaborative resource to support and expedite the development of high-quality tests. We encourage all institutions and researchers working in this field to make use of this important resource.

Stefano Ongarello ${ }^{\square}$,

Marta Fernández Suárez and Fay Betsou

FIND, the global alliance for diagnostics, Geneva, Switzerland.

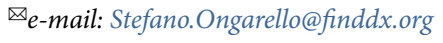

Published online: 22 December 2021

https://doi.org/10.1038/s41587-021-01168-z

\section{References}

1. World Health Organization. https://www.who.int/news/item/ 28-09-2020-global-partnership-to-make-available-120-millionaffordable-quality-covid-19-rapid-tests-for-low--and-middleincome-countries (2020).

2. van Draanen, J. et al. Biopreserv. Biobank. 15, 203-210 (2017).

3. Sedyaningsih, E. R. et al. Ann. Acad. Med. Singap. 37, 482-488 (2008)

4. Freudenthal, E. The Telegraph, https://www.telegraph.co.uk/ global-health/science-and-disease/ebolas-lost-blood-rowsamples-flown-africa-big-pharma-set-cash/ (February 2019)

5. de Souza, D. K. et al. PLoS Negl. Trop. Dis. 14, e0008587 (2020).

6. Petway, M. et al. Public Health Rep. 134 (Suppl), 53S-57S (2019).

7. Kelly-Cirino, C. D. et al. BMJ Glob. Health 4, e001179 (2019).

8. Coppola, L. et al. J. Transl. Med. 17, 172 (2019).

9. Zawati, M. H. et al. Biopreserv. Biobank. 16, 171-178 (2018).

Acknowledgements

The DxConnect Virtual Biobank is hosted by FIND with support from the Bill \& Melinda Gates Foundation and Unitaid. The software platform has been developed in collaboration with the Digital Research Service department at the University of Nottingham. The authors thank Anna Mantsoki and Imane El Idrissi in the FIND biobank team and Infinity Communications for manuscript assistance in line with Good Publication Practice 3.

Competing interests

S.O., M.F.S. and F.B. are employed by FIND, the global alliance for diagnostics. 